\title{
How well can infrared sounders observe the atmosphere and surface through clouds?
}

\author{
Daniel K. Zhou*1, Allen M. Larar ${ }^{1}$, Xu Liu ${ }^{1}$, William L. Smith ${ }^{2,3}$, \\ L. Larrabee Strow ${ }^{4}$, and Ping Yang ${ }^{5}$ \\ ${ }^{1}$ NASA Langley Research Center, Hampton, VA, USA \\ ${ }^{2}$ Hampton University, Hampton, VA, USA \\ ${ }^{3}$ University of Wisconsin-Madison, Madison, WI, USA \\ ${ }^{4}$ University of Maryland Baltimore County, Baltimore, MD, USA \\ ${ }^{5}$ Texas A\&M University, College Station, TX, USA
}

\begin{abstract}
Infrared sounders, such as the Atmospheric Infrared Sounder (AIRS), the Infrared Atmospheric Sounding Interferometer (IASI), and the Cross-track Infrared sounder (CrIS), have a cloud-impenetrable disadvantage in observing the atmosphere and surface under opaque cloudy conditions. However, recent studies indicate that hyperspectral, infrared sounders have the ability to detect cloud effective-optical and microphysical properties and to penetrate optically thin clouds in observing the atmosphere and surface to a certain degree. We have developed a retrieval scheme dealing with atmospheric conditions with cloud presence. This scheme can be used to analyze the retrieval accuracy of atmospheric and surface parameters under clear and cloudy conditions. In this paper, we present the surface emissivity results derived from IASI global measurements under both clear and cloudy conditions. The accuracy of surface emissivity derived under cloudy conditions is statistically estimated in comparison with those derived under clear sky conditions. The retrieval error caused by the clouds is shown as a function of cloud optical depth, which helps us to understand how well infrared sounders can observe the atmosphere and surface through clouds.
\end{abstract}

Keywords: Remote sensing, ultraspectral instrument, inversion, clouds, land surface emissivity.

\section{INTRODUCTION}

Surface spectral emissivity (SSE or $\varepsilon_{v}$ ) and surface skin temperature $\left(T_{s}\right)$ from current and future operational satellites can and will reveal critical information about the Earth's ecosystem and land surface type properties. The Earth Observing System (EOS) Aqua satellite, carrying the Atmospheric InfraRed Sounder (AIRS), was launched on May 4, 2002. Satellite ultraspectral data (such as that from AIRS) have been shown to be significant for atmospheric research and monitoring the Earth's environment ${ }^{1}$. The Infrared Atmospheric Sounding Interferometer (IASI) ${ }^{2}$, aboard the Metop-A satellite ${ }^{3}$ launched on October 19, 2006, is the first of the advanced ultra-spectral resolution temperature, humidity, and trace gas sounding instruments being flown as part of the Initial Joint Polar System (IJPS) of POES (U.S. Polar Orbiting Environmental Satellites) and EPS (EUMESAT Polar System) programs. It is the precursor of the Joint Polar System (JPS) of the National Polar-orbiting Operational Environmental Satellite System (NPOESS) and EPS operational satellites for the purpose of improved weather forecasting, climate monitoring, and air quality observation. Future satellite instruments, such as the second and third units of IASI on the European Metop-B and -C satellites, the Cross-track Infrared Sounder (CrIS) on the NPOESS Preparatory Project (NPP), and the series of NPOESS satellites, will continue to provide ultraspectral and/or hyperspectral infrared (IR) radiance observations that will be used for longterm monitoring of the Earth's environment and climate change. Long-term and large-scale observations needed for global climate change monitoring and other research can only be supplied by space-based remote sensing ${ }^{4}$. SSE retrieved from satellite ultraspectral IR measurements is crucial for achieving other highly accurate retrieved parameters, such as the surface skin temperature retrieved from other satellite broad-band measurements, the assimilation of IR radiances in numerical weather prediction models, climate monitoring, and studies of the radiation budget for the Earth system.

*daniel.k.zhou@,nasa.gov, Tel: 757-864-5663 
Several algorithms have been developed to derive IR emissivity from satellite measurements ${ }^{5-12}$. Recent work ${ }^{13}$ based on Zhou et al. ${ }^{5}$ has been published. The work describes an improved multi-stage retrieval algorithm with an emphasis on the regression-training database and an SSE constraining function used in the retrieval with samples demonstrating SSE retrieved from different surface types Rapidly retrieved SSE using IASI satellite global measurements in conjunction with some initial retrieval analyses are demonstrated. Initial validation for retrieved SSE is conducted with laboratory measurements from the Namib and Kalahari deserts. Global SSE with a spatial resolution of $0.5^{\circ} \times 0.5^{\circ}$ in latitudelongitude are produced with a seasonal variation, indicating seasonal diversity of global land SSE.

As the retrieval is performed over all measurements, including during both clear-sky and cloudy conditions and when the temporal variation of the surface emissivity is subtle within a short period of time, the retrieved surface emissivity is used to build up a climatology dataset to estimate the cloud effects on geophysical parameter retrieval accuracy. This dataset is essential in helping us understand that how well infrared sounders can observe the atmosphere and surface through clouds. In this paper, four years of IASI measurements derived from surface emissivity spectra are used to present a statistical analysis on the retrieval accuracy of surface emissivity under different cloud categories - mainly the effective cloud optical depth.

\section{RETRIEVAL ALGORITHM}

\subsection{Radiative transfer equation and radiance calculation}

Following the terminology used by Strow et al. ${ }^{14}$, the channel radiance $R_{v}$ leaving the top of a nonscattering, clear atmosphere at a certain wavenumber $v$ can be computed via

$$
\begin{aligned}
R_{v}= & \varepsilon_{v} B_{v}\left(T_{s}\right) \tau_{v}\left(p_{s} \rightarrow 0, \theta_{s a t}\right)+\int_{p_{s}}^{0} B_{v}[T(p)] \frac{d \tau_{v}\left(p \rightarrow 0, \theta_{s a t}\right)}{d p} d p \\
& +F_{v}^{d} \rho_{v}^{t} \tau_{v}\left(p_{s} \rightarrow 0, \theta_{s a t}\right)+\frac{H_{v}}{\sec \left(\theta_{\text {sun }}\right)} \tau_{v}\left(0 \rightarrow p_{s}, \theta_{\text {sun }}\right) \rho_{v}^{s} \tau_{v}\left(p_{s} \rightarrow 0, \theta_{\text {sat }}\right),
\end{aligned}
$$

where $\varepsilon_{v}$ is SSE, $B_{v}$ is the Planck function, $\tau_{v}$ is the atmospheric layer-to-space transmittance, $p_{s}$ is surface pressure, $T_{s}$ is the surface skin temperature, $T(p)$ is atmospheric temperature at pressure $p, \rho_{v}^{t}$ is the reflectance of the down-welling thermal flux $F_{v}^{d}$ by the surface, $H_{v}$ is the solar irradiance incident at the top of the atmosphere, $\rho_{v}^{s}$ is the solar reflectance of the surface, and $\theta_{\text {sat }}$ and $\theta_{\text {sun }}$ are satellite and sun zenith angles, respectively. The surface is assumed to be Lambertian, and reflectivity is assumed to be a function of surface emissivity, such as $\rho_{v}^{t}=\left(1-\varepsilon_{v}\right)$ and $\rho_{v}^{s}=\left(1-\varepsilon_{v}\right) / \pi$. Eq. (1) can then be re-written as follows:

$$
\begin{aligned}
R_{v}= & {\left[\int_{p_{s}}^{0} B_{v}[T(p)] \frac{d \tau_{v}\left(p \rightarrow 0, \theta_{\text {sat }}\right)}{d p} d p+F_{v}^{d} \tau_{v}\left(p_{s} \rightarrow 0, \theta_{\text {sat }}\right)\right.} \\
& \left.+\frac{H_{v}}{\sec \left(\theta_{\text {sun }}\right) \pi} \tau_{v}\left(0 \rightarrow p_{s}, \theta_{\text {sun }}\right) \tau_{v}\left(p_{s} \rightarrow 0, \theta_{\text {sat }}\right)\right]+ \\
& {\left[B_{v}\left(T_{s}\right) \tau_{v}\left(p_{s} \rightarrow 0, \theta_{\text {sat }}\right)-F_{v}{ }^{d} \tau_{v}\left(p_{s} \rightarrow 0, \theta_{\text {sat }}\right)\right.} \\
& \left.-\frac{H_{v}}{\sec \left(\theta_{\text {sun }}\right) \pi} \tau_{v}\left(0 \rightarrow p_{s}, \theta_{\text {sun }}\right) \tau_{v}\left(p_{s} \rightarrow 0, \theta_{\text {sat }}\right)\right] \varepsilon_{v} \\
= & K_{1}+K_{2} \varepsilon_{v},
\end{aligned}
$$

where 


$$
\begin{aligned}
K_{1}= & {\left[\int_{p_{s}}^{0} B_{v}[T(p)] \frac{d \tau_{v}\left(p \rightarrow 0, \theta_{\text {sat }}\right)}{d p} d p+F_{v}^{d} \tau_{v}\left(p_{s} \rightarrow 0, \theta_{\text {sat }}\right)\right.} \\
& \left.+\frac{H_{v}}{\sec \left(\theta_{\text {sun }}\right) \pi} \tau_{v}\left(0 \rightarrow p_{s}, \theta_{\text {sun }}\right) \tau_{v}\left(p_{s} \rightarrow 0, \theta_{\text {sat }}\right)\right], \\
K_{2}= & {\left[B_{v}\left(T_{s}\right) \tau_{v}\left(p_{s} \rightarrow 0, \theta_{\text {sat }}\right)-F_{v}^{d} \tau_{v}\left(p_{s} \rightarrow 0, \theta_{\text {sat }}\right)\right.} \\
& \left.\quad-\frac{H_{v}}{\sec \left(\theta_{\text {sun }}\right) \pi} \tau_{v}\left(0 \rightarrow p_{s}, \theta_{\text {sun }}\right) \tau_{v}\left(p_{s} \rightarrow 0, \theta_{\text {sat }}\right)\right] .
\end{aligned}
$$

Radiance is a linear function of the emissivity. It is assumed that the other parameters in the coefficients $K_{1}$ and $K_{2}$ are retrievable with ultraspectral radiance measurements.

Under cloudy conditions, the top of atmosphere radiance for an atmosphere containing a single cloud layer is expressed by the monochromatic, radiative-transfer equation at those frequencies (note that the wavenumber $v$ is omitted here) ${ }^{15}$,

$$
R=R_{0} F_{T} \tau_{t c}+R_{c} \tau_{t c}+R_{1}+R_{1}^{\downarrow} F_{R} \tau_{t c},
$$

where $R$ is the total upwelling spectral radiance at the top of atmosphere. $F_{T}$ and $F_{R}$ are the cloud transmission (including both direct and diffuse parts) and reflection functions, respectively. The terms $R_{0}, R_{c}, R_{1}, R_{1}^{\downarrow}$, and $\tau_{t c}$ are upwelling emission below the cloud, emission from the cloud, upwelling emission above the cloud, downwelling emission above the cloud, and the transmittance between the cloud level and the top of the atmosphere, respectively. Detailed expressions for these terms are described by Zhou et al. ${ }^{15}$. If the components of solar radiation and reflectivity do not affect the channels used in the retrieval, the SSE is still linear with respect to the radiance under cloudy conditions. Under clear-sky conditions, the surface (or window) channel radiance $R_{v}$ is sensitive to surface skin temperature and SSE, and the SSE $\left(\varepsilon_{v}\right)$ is a function of wavenumber. With ultraspectral radiance measurements, SSE should be retrieved with a linear retrieval model. In other words, linear-regression-retrieved SSE should be as accurate as physically retrieved SSE if the regression-training database is optimal in the sense of representing actual SSE from a linear combination of the database conditions. In this paper, we present regression retrieved SSE with improved regression training and coefficients.

Within this study, we use IASI measurements to derive global land surface properties to estimate the cloud effects on the emissivity. IASI is a Michelson interferometer with spectral coverage between 3.62 and $15.5 \mu \mathrm{m}$. At nadir, the instrument collects data at intervals of $25 \mathrm{~km}$ along track, each sample having four pixels and each pixel having a maximum spatial diameter of about $12 \mathrm{~km}$. The IASI fast transmittance model used herein is a combination of the Stand-alone AIRS Radiative Transfer Algorithm (SARTA) ${ }^{14}$ Version 1.07 and the physically-based cloud radiative transfer model (RTM) based on the DIScrete Ordinate Radiative Transfer (DISORT) ${ }^{16}$ calculations performed for a wide variety of cloud microphysical properties ${ }^{17}$. The retrieval algorithm used for IASI is based on that first developed and demonstrated with NAST-I using a regional-seasonal EOF regression-training database ${ }^{5,18}$.

\subsection{Training database for regression}

Our purpose of using a training database is to generate regression coefficients for global IASI retrievals; therefore, a globally representative training database with a large diversity is required. The regression-training database consists of profiles obtained from the SeeBor database ${ }^{19}$, SSE from a set of laboratory measured SSE for a wide variety of surface types $^{20}$, and cloud microphysical properties from a parameterization based on a balloon and aircraft cloud microphysical database $^{21}$. A random number generator is used to specify cloud-visible optical depth equally distributed within a prespecified range that is sensitive to IR RTM calculations. A parameterization based on a balloon and aircraft cloud microphysical database is used to specify cloud effective particle diameter $\left(D_{e}\right)$ from the cloud optical depth $\left(\tau_{c l d}\right)^{15,22}$. A simulated retrieval analysis has been performed to assess retrieval accuracy including surface parameters using the SeeBor database. Based on our analyses, a few minor modifications are made to the training database in order to provide improved retrievals (e.g., surface parameters). Land surface parameter retrieval accuracies are modified to obtain a more diverse contrast of surface skin and surface air temperature, especially over extreme hot and/or cold (e.g., desert and/or polar) regions. Land surface skin temperature in the SeeBor training database is replaced by 


$$
T_{s}=T_{a}+T_{\delta}
$$

where $T_{a}$ and $T_{s}$ are surface air and skin temperature, respectively, and $T_{\delta}$ is a random number generated value with a mean of $0 \mathrm{~K}$ and a standard deviation (STD) of $3 \mathrm{~K}$ over water and $10 \mathrm{~K}$ over land.

SSE is also replaced by a randomly selected laboratory measured SSE from a wide variety of surface types ${ }^{20}$. The SSE function,

$$
F\left(\varepsilon_{v}\right)=\log \left[\log \left(\varepsilon^{\mathrm{HB}}-\varepsilon^{\mathrm{LB}}+0.005\right)-\log \left(\varepsilon^{\mathrm{HB}}-\varepsilon_{v}\right)\right],
$$

is introduced here for usage in the training and retrieval algorithm instead of SSE itself in order to constrain the retrieved SSE within a boundary between $\varepsilon^{\mathrm{LB}}$ (i.e., 0.5 ) and $\varepsilon^{\mathrm{HB}}$ (i.e., 0.995) being preset to the best of our knowledge for surface emissivities. These SSE functional spectra $F(\varepsilon)$ are represented by their nine highest-ranking EOF amplitudes

$$
A_{i}^{F}=\sum_{j=1}^{n c h} F_{j}(\varepsilon) \varphi_{j i}^{F}
$$

of the eigenvectors $\varphi^{F}$ generated with a set of SSE functional spectra $F(\varepsilon)$ computed with the laboratory measured SSE database, where $n c h$ is the number of channels used in the retrieval, and $i$ is the index number of EOF amplitudes. In order to minimize the compression error, the nine highest-ranking eigenvectors are used to compress SSE functional spectra $F(\varepsilon)$ in the retrieval. An equivalent SSE STDE from this compression is below 0.006 with a mean error near zero.

\subsection{Regression retrieval}

An improved regression algorithm has been introduced based on our previous version ${ }^{23}$. In addition to the modifications made on the training database mentioned above, several modifications are made to the regression retrieval. These modifications are made through retrieval analyses with simulations and real data retrievals. The new scheme includes more regression channels in the window regions (i.e., near transparent regions of the spectrum). Solar radiation has a small, but significant, impact on some of the selected channel radiances; solar radiation is calculated in the RTM using the solar zenith angle as a predictor. A total of 5410 channels are currently used for regression, and the 1766 channels are used for the variational retrieval. Standard EOF regression equations are expressed as

$$
\begin{gathered}
A_{i}=\sum_{j=1}^{n c} R_{j} \varphi_{j i}, \\
S_{j}=\sum_{i=1}^{n-1} K_{j i} A_{i}+K_{j n} P_{s}=\sum_{i=1}^{n-1} K_{j i}\left(\sum_{l=1}^{n c} R_{l} \varphi_{l i}\right)+K_{j n} P_{s}
\end{gathered}
$$

where $\boldsymbol{A}$ and $\boldsymbol{\varphi}$ are radiance EOF amplitudes (i.e., PC scores) and radiance eigenvectors, respectively. The eigenvectors are generated with a set of synthetic radiances $\boldsymbol{R} . \boldsymbol{K}$ is the regression coefficient generated with a set of synthetic radiances $\boldsymbol{R}$ in the EOF domain (i.e., $\boldsymbol{A}$ ) and the associated state vector $\boldsymbol{S}$ (i.e., the training dataset), and $P_{s}$ is surface pressure. In our regression retrieval, a state vector includes atmospheric profiles (i.e., temperature, moisture, ozone, and carbon monoxide), cloud parameters (i.e., cloud top height $H_{c}$, optical depth $\tau_{c l d}$, particle size $D_{e}$, and cloud phase $\phi$ ), surface skin temperature $T_{s}$, and surface SSE functional amplitudes $\boldsymbol{A}^{F}$.

The regression coefficients are classified with respect to cloud-free, cloudy, and all combined (mixed) conditions. A multi-step regression approach uses cloudy and clear coefficients for cloud and clear radiances. Cloud detection criteria in the regression are re-investigated in order to provide accurate "cloud-free," single field-of-view (SFOV) measurements. The first-stage involves mixed (i.e., clear and cloudy) regression. The second-stage will be either clear or cloudy regression depending on the cloud detection criteria that are based on first-stage retrieved cloud parameters. 
The second-stage will use clear regression coefficients when the first retrieval satisfies these two criteria: (1) $\phi \leq 0.8$ and (2) $\tau_{c l d} \leq 0.007$, where $\phi=0,1$, and 2 are for clear-sky conditions, ice clouds, and water clouds, respectively. Otherwise, the second stage will use cloudy regression coefficients. When the second stage uses cloudy regression, an additional clear regression will be performed if one of the two following criteria is met from the second-stage cloudy retrieval: (1) $\left(H_{c}-H_{s}\right) \leq 2.0 \mathrm{~km}$ with $\tau_{c l d} \leq 0.8$, and (2) $\left(H_{c}-H_{s}\right)>2.0 \mathrm{~km}$ with $\tau_{c l d} \leq 0.2$ (where $H_{s}$ is surface height). These criteria are chosen from empirical experience based on global retrieval analysis with this training database. Actually, it should be explained that the so-called "clear-sky" cases from this analysis are "cloud-undetected." The sky can be either clear or containing a minimal cloud fraction barely interfering (and hence "undetected") with the retrieval.

When $\boldsymbol{A}^{F}$ is retrieved from measured radiance as part of the state vector, the $\boldsymbol{F}(\varepsilon)$ are then computed with the eigenvectors covering the entire IASI spectral region. Eqs. (7) and (8) can be re-written as

$$
\begin{aligned}
\varepsilon_{j} & =\varepsilon^{\mathrm{HB}}-\exp \left[\log \left(\varepsilon^{\mathrm{HB}}-\varepsilon^{\mathrm{LB}}+0.005\right)-\exp \left(F_{j}\right)\right] \\
& =\varepsilon^{\mathrm{HB}}-\exp \left[\log \left(\varepsilon^{\mathrm{HB}}-\varepsilon^{\mathrm{LB}}+0.005\right)-\exp \left(\sum_{i=1}^{9} \varphi_{j i}^{F} A_{i}^{F}\right)\right] .
\end{aligned}
$$

In other words, the SSE is derived for all IASI channels even though some channels are not selected for retrieval. However, the channel selection does affect the quality of the retrievals; therefore, a large set of representative channels covering a large portion of the spectral range is necessary to retrieve SSE.

Detailed algorithm performance, analysis, emissivity evaluation and initial validation, as well as emissivity atlas with temporal variation can be found in Zhou et al. ${ }^{13}$

\section{EMISSIVITY DATASETS AND ANAYSIS}

The retrievals have gone through quality control (i.e., retrieval consistency check) based on a standard deviation of the difference between the measured and retrieval-simulated brightness temperature over the spectrum of physical retrieval channels' spectral-radiance fitting residual. The retrieval fitting residual is strongly dependent on the accuracy of retrieval parameters. IASI retrievals include effective cloud microphysical properties, surface parameters (under clear conditions), and atmospheric temperature and moisture profiles. Cloud microphysical parameters retrieved from the IR ultraspectral data are "effective" in the sense that they depend on instrument characteristics (e.g., FOV size) and spectral dependence of the infrared sensitivity of the cloud property. For instance, observed IR radiance has a limited sensitivity variation of cloud optical depth for large values, i.e., a retrieved "effective" optical depth of an opaque can be quite different from the real optical depth of the cloud. Although the cloud retrieval algorithm has been evaluated with aircraft data (e.g., dropsondes and Cloud Physics Lidar) from the THORPEX Atlantic Regional Campaign (ATReC) ${ }^{7}$, more validations for diverse cloud conditions are desired to give definitive conclusions regarding the retrieval accuracy. Retrieval evaluation and validation have been performed and found elsewhere ${ }^{13}$.

Global IASI measurements since June 2007 are used to produce retrievals that are then used to generate emissivity climatology datasets. Here in this study, we use July 2007 to 2010 (four years) data to make emissivity datasets under clear-sky conditions and cloudy conditions with three cloud categories, depending on retrieved effective cloud optical depth. These three categories are (1) optical depth less than 0.5 , (2) greater then 0.5 and less than 1.0, and (3) greater than 1 . The emissivity spectra retrieved under cloudy conditions are affected by the clouds; their deviation from those retrieved from clear-sky measurements implies how well the information is obtained from the clouds and below. We take advantage of the minimal temporal-variation of surface emissivity and use the statistical analysis on the emissivity retrieved from clear and cloudy conditions to reveal how well infrared sounders observe the atmosphere and surface through clouds. For example, regional emissivity atlases at the selected wave numbers are plotted in figure 1 for clearsky and three categories of clouds. As the retrieved cloud optical depth increases, the emissivity under cloudy conditions deviates further from that of clear-sky conditions. The cloud effects on the emissivity retrievals can be used to estimate the error introduced to other retrieved geophysical parameters, such as surface temperature and atmospheric profiles of trace species. Figure 1 shows the region of Asia, while figure 2 shows the region of Northern America for clarity. Other regions or global atlas are also used for analysis with similar results but not shown here. The degree of emissivity 
deviation due to the cloud effectiveness is not necessarily the same at different wavenumbers; somehow, the emissivity feature is there even if it is under opaque clouds where emissivity could not be physically retrieved because the regression retrieval uses a training database that constrains the results. It is clear that when the cloud optical depth is greater than 1.0, the emissivity is not retrieved correctly as shown by its spatial variation and absolute values.

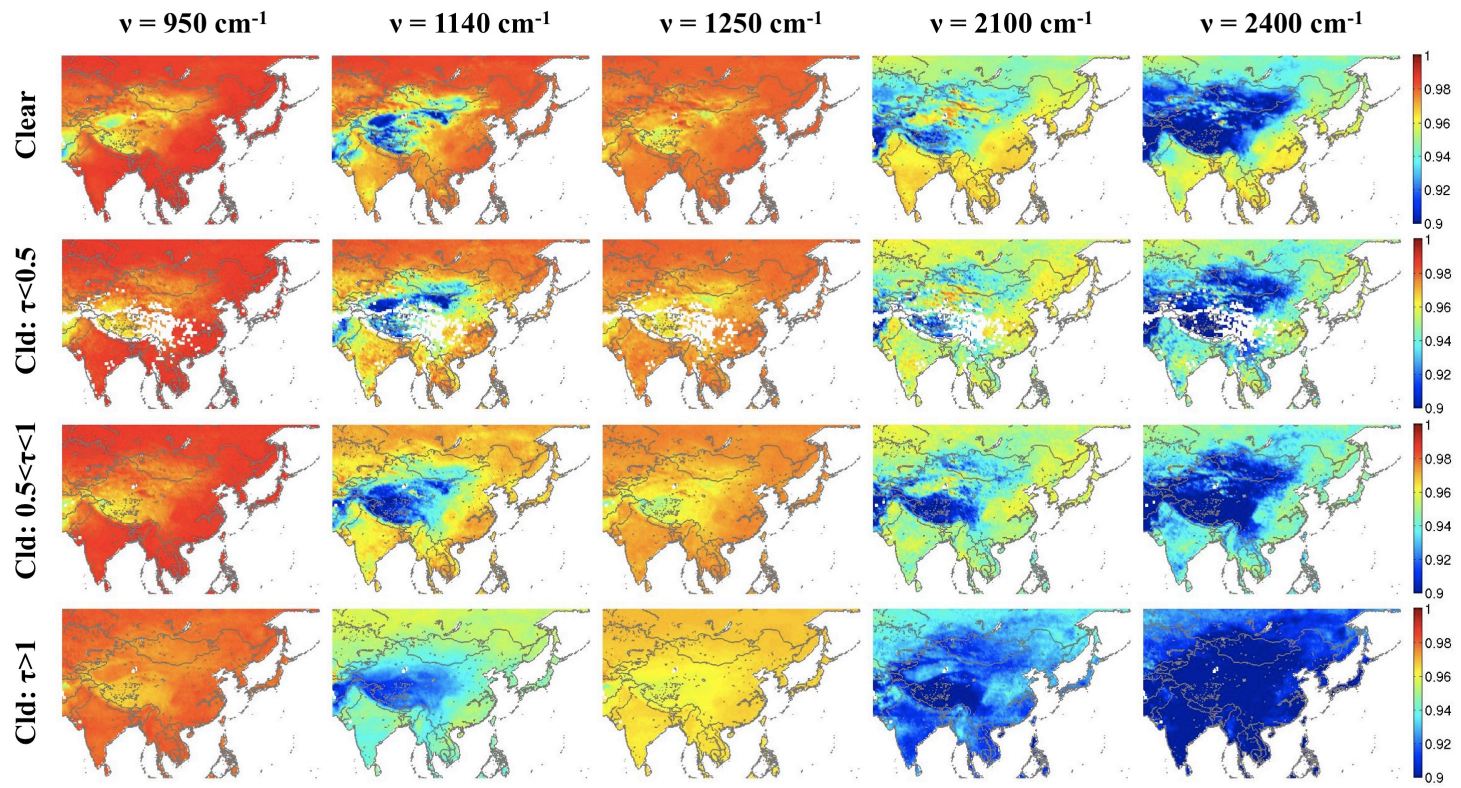

Figure 1. Asian regional atlas table shows emissivity at five selected wavenumbers (in column) versus clear and cloudy conditions (in row). As the retrieved cloud optical depth increases, the emissivity under cloudy conditions deviated more from that of clear conditions.

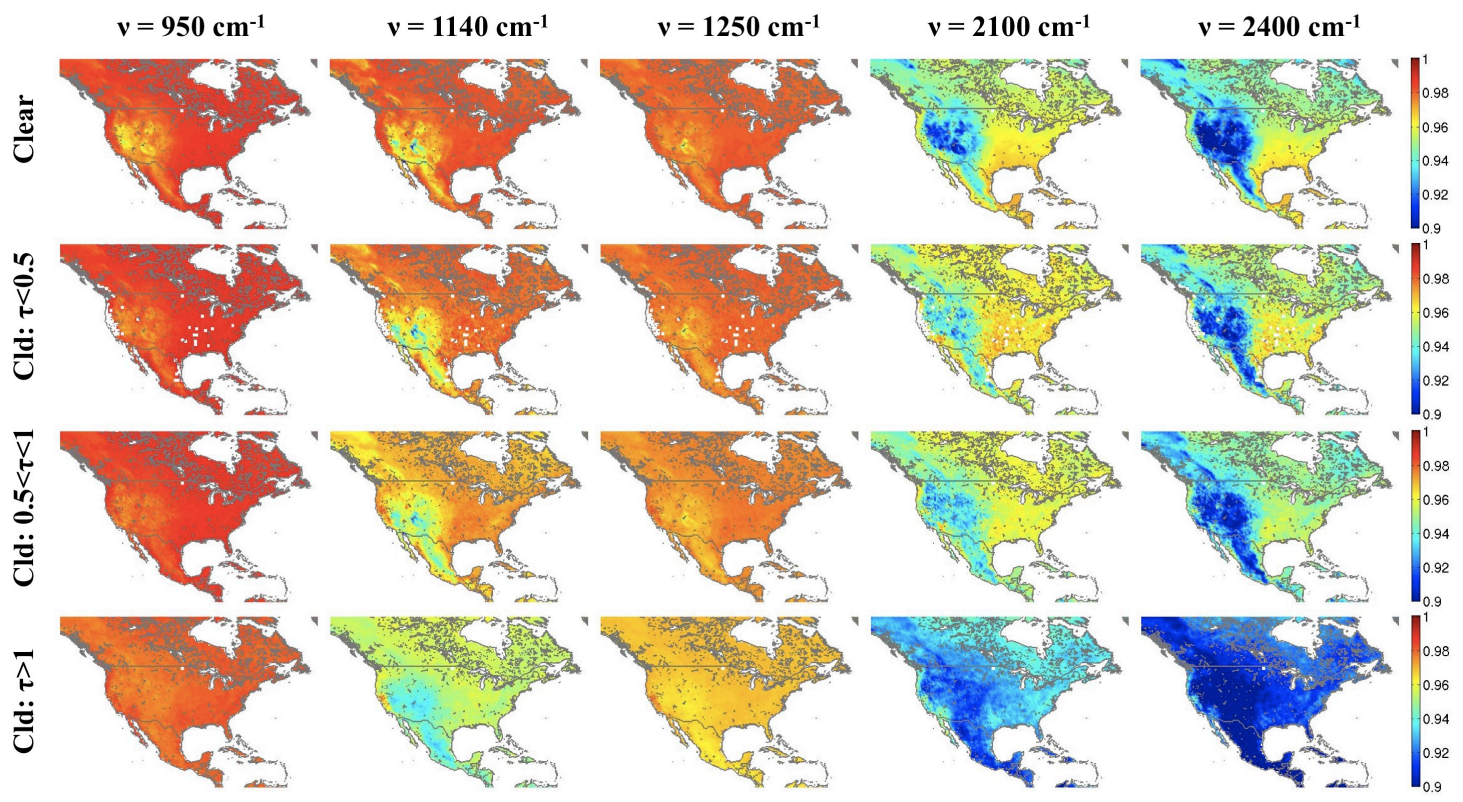

Figure 2. This is the same as figure 1, but in the region of Northern America. 
It is expected that clouds affect emissivity retrieval further in the short wave region than that in the long wave region because the nature of the cloud radiation and/or reflection is more sensitive in the short wave region. Nevertheless, in order to see the error in the whole spectral domain associated with the data plotted in figure 1, figure 3 shows the mean emissivity and their standard deviation (indicating spatial variation) under clear and cloudy conditions, as well as the difference between clear and cloudy results. It is difficult to tell an exact error of an individual retrieval from this regional statistical result, but it indicates the relative error on each spectral channel. It is noted that some channels seem unaffected by the clouds because they are opaque channels that are not sensitive to the surface. Similar results from the region of Northern America are also shown in figure 4.

In response to our question, "how well can infrared sounders observe the atmosphere and surface through clouds," we believe it depends on the accuracy we need. To obtain the retrieval accuracy for geophysical parameters very similar to that from the clear-sky conditions, effective cloud optical depth needs to be less than 0.5 . When cloud optical depth the between 0.5 and 1.0, the retrieval error is clearly noticeable. However, when the cloud optical depth is greater than 1.0, the retrieval is not acceptable as it gives wrong information, as shown in figures 1 and 2.
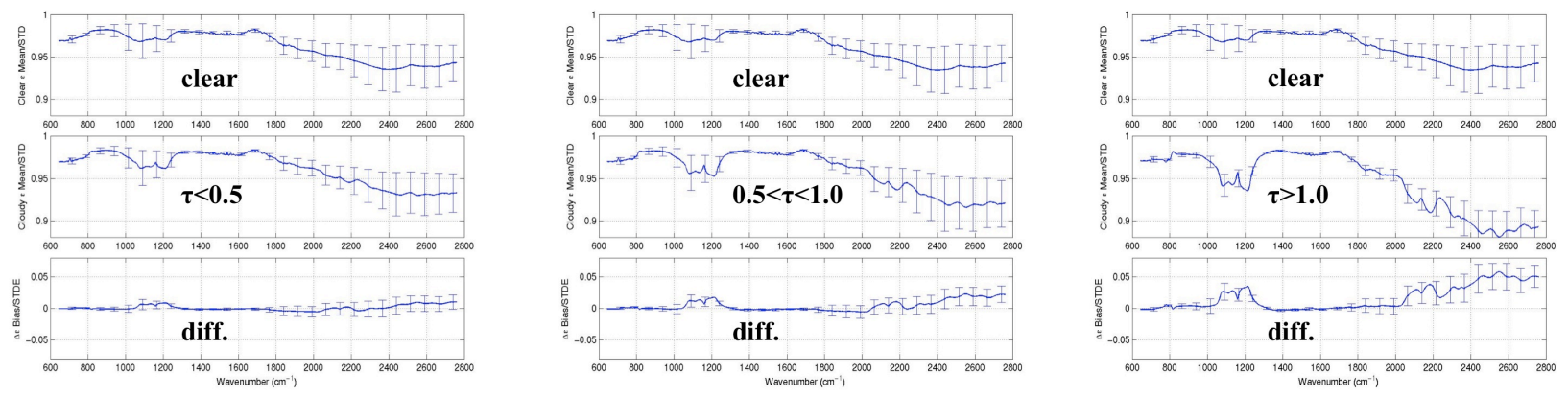

Figure 3. Asian regional statistical results associated with figure 1 show the mean (curve) and standard deviation (vertical bars). The top panels are from the clear conditions, the middle panels are from the cloudy conditions with three cloud categories, and the bottom panels are the difference between clear and cloudy.
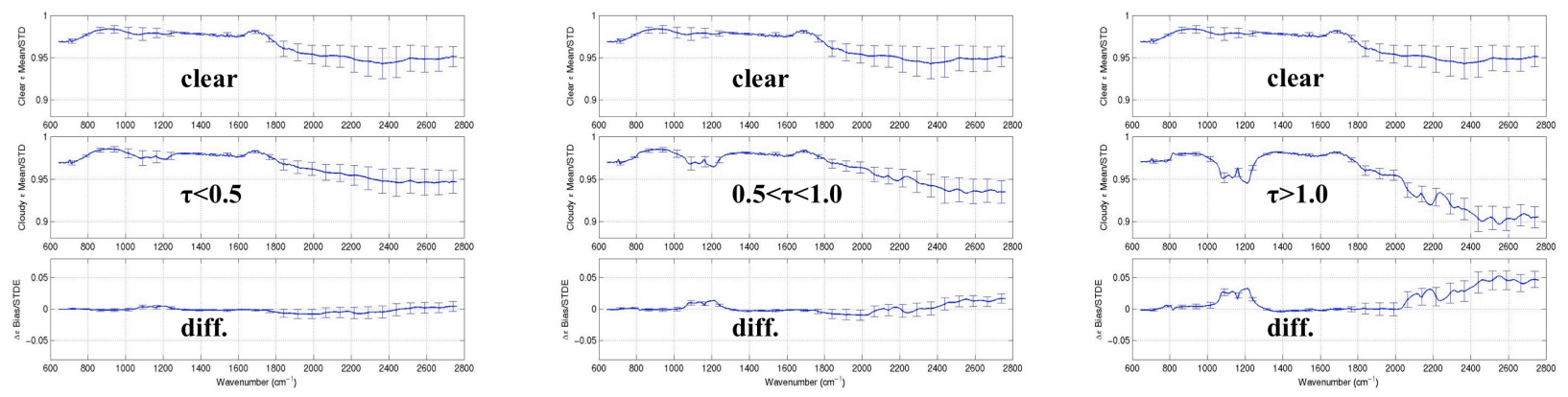

Figure 4. As same as figure 3 but in the region of Northern America associated with figure 2.

\section{SUMMARY}

A state-of-the-art retrieval algorithm with the ability to account for all-weather conditions has been developed and applied to IASI radiance measurements. SSE is retrieved from satellite IR ultraspectral data to capture different land, surface-type properties that contain useful information on the state of the terrestrial ecosystem and reflect on the biosphere's response to proximal climatic factors (such as temperature and rainfall) and human activities. Operational, global, satellite data such as observations from IASI and CrIS are, and will be, available for deriving these kinds of products. Based on our analysis, in order to obtain the retrieval accuracy for geophysical parameters very similar to that from the clear-sky conditions, effective cloud optical depth needs to be less than 0.5 . When cloud optical depth is 
between 0.5 and 1.0, the retrieval error is clearly noticeable. However, then the cloud optical depth is greater than 1.0, the retrieval will not be not acceptable as it gives wrong information. This can apply to other retrieved geophysical parameters since they are strongly connected to the surface emissivity accuracy.

\section{ACKNOWLEDGEMENTS}

This research is supported by NASA Langley Research Center and NASA Headquarters. The global training database SeeBor was provided by Eva Borbas from the Space Science and Engineering Center of the University of Wisconsin. IASI was developed and built under the responsibility of the Centre National d'Etudes Spatiales (CNES). It is flown aboard the Metop satellites as part of the EUMETSAT Polar System. The IASI L1C data are received through the Unified Meteorological Archival and Retrieval Facility (UMARF) of EUMETSAT.

\section{REFERENCES}

1. C. Chahine and Co-authors, "AIRS: improving weather forecasting and providing new insights into climate," B. Am. Meteorol. Soc., vol. 87, no. 7, pp. 911-926, Jul. 2006.

2. D. Blumstein, G. Chalon, T. Carlier, C. Buil, P. Hebert, T. Maciaszek, G. Ponce, and T. Phulpin, "IASI instrument: technical overview and measured performances," SPIE Proc., vol. 5543, pp. 196-207, Nov. 2004.

3. D. K. Klaes, M. Cohen, Y. Buhler, P. Schlüssel, R. Munro, J.-P. Luntama, A. Von Engeln, E. Ó. Clerigh, H. Bonekamp, J. Ackermann, J. Schmetz, "An introduction to the EUMETSAT Polar System", B. Am. Meteorol. Soc., vol. 88, no. 7, pp. 1085-1096, Jul. 2007.

4. S. Schädlich, F. M. Göttsche, F.-S. Olesen, "Influence of land surface parameters and atmosphere on METEOSAT brightness temperature and generation of land surface temperature maps by temporally and spatially interpolating atmospheric correction," Remote Sens. Environ., 75, no. 1, pp. 39-46, Jan. 2001.

5. D. K. Zhou, W. L. Smith, J. Li, H. B. Howell, G. W. Cantwell, A. M. Larar, R. O. Knuteson, D. C. Tobin, H. E. Revercomb, and S. A. Mango, "Thermodynamic product retrieval methodology for NAST-I and validation," App. Opt., vol. 41, no. 33, pp. 6957-6967, Nov. 2002.

6. C. C. Borel, "Surface emissivity and temperature retrieval for a hyperspectral sensor," Geoscience and Remote Sensing Symposium Proceedings, vol. 1, pp. 546-549, 1998.

7. E. Péquignot, A. Chédin, and N. A. Scott, "Infrared Continental Surface Emissivity Spectra Retrieved from AIRS Hyperspectral Sensor," J. Appl. Meteorol. Climatol., vol. 47, no. 6, pp. 1619-1633, June 2008.

8. R. O. Knuteson, F. A. Best, D. H. DeSlover, B. J. Osborne, H. E. Revercomb, W. L. Smith, Sr., "Infrared land surface remote sensing using high spectral resolution aircraft observations," Advances In Space Research, vol. 33, no. 7, pp. 1114-1119, Dec. 2003.

9. J. Susskind and J. Blaisdell, "Improved surface parameter retrievals using AIRS/AMSU data", Proc. SPIE, vol. 6966, pp. 10-12, Apr. 2008.

10. J. Li, J. Li, E. Weize, and D. K. Zhou, "Physical retrieval of surface emissivity spectrum from hyperspectral infrared radiances," Geophys. Res. Lett., vol. 34, no. L16, pp. 812.1-812.6, 2007.

11. L. Zhou, M. Goldberg, C. Barnet, Z. Cheng, F. Sun, W. Wolf, T. King, X. Liu, H. Sun, and M. Divakarla, "Regression of surface spectral emissivity from hyperspectral instruments," IEEE Trans. Geosci. Remote Sens., vol. 46, no. 2, pp. 328-333, Feb. 2008

12. X. Liu, D. K. Zhou, A. M. Larar, W. L. Smith, P. Schlüssel, S. M. Newman, J. P. Taylor, and W. Wu, "Retrieval of atmospheric profiles and cloud properties from IASI spectra using super-channels," Atmos. Chem. Phys., vol. 9, pp. 9121-9142, Dec. 2009.

13. D. K. Zhou, A. M. Larar, X. Liu, W. L. Smith, L. L. Strow, P. Yang, P. Schlüssel, and X. Calbet, "Global land surface emissivity retrieved from satellite ultraspectral IR measurements," IEEE Trans. Geosci. Remote Sensing, in press, 2010.

14. L. L. Strow, S. E. Hannon, S. De Souza-Machado, H. E. Motteler, and D. Tobin, "An overview of the AIRS radiative transfer model," IEEE Trans. Geosci. Remote Sens., vol. 41, no. 2, pp. 303-313, Feb. 2003.

15. D. K. Zhou, W. L. Smith, Sr., X. Liu, A. M. Larar, S. A. Mango, and H.-L. Huang, "Physically retrieving cloud and thermodynamic parameters from ultraspectral IR measurements," J. Atmos. Sci., vol. 64, no. 3, pp. 969-982, Mar. 2007. 
16. K. Stamnes, S.-C. Tsay, W. Wiscombe, and K. Jayaweera, "Numerically stable algorithm for discrete-ordinatemethod radiative transfer in multiple scattering and emitting media," App. Opt., 27, no. 12, pp. 2502-2509, Jun. 1988.

17. P. Yang, B. C. Gao, B. A. Baum, Y. Hu, W. J. Wiscombe, S.-C. Tsay, D. M. Winker, and S. L. Nasiri, "Radiative Properties of cirrus clouds in the infrared $(8-13 \mu \mathrm{m})$ spectral region,” J. Quant. Spectros. Radiat. Transfer, vol. 70, no. 4, pp. 473-504, Aug. 2001.

18. D. K. Zhou, A. L. Larar, W. L. Smith, and X. Liu, "Surface emissivity effects on thermodynamic retrieval of IR spectral radiance,” Proc. SPIE, vol. 6405, pp. 1H1-1H8, Dec. 2006.

19. S. W. Seemann, E. E. Borbas, R. O. Knuteson, G. R. Stephenson, and H.-L. Huang, "Development of a global infrared land surface emissivity database for application to clear-sky sounding retrievals from multi-spectral satellite radiance measurements," J. Appl. Meteorol. Climatol., vol. 47, no. 1, pp. 108-123, Jan. 2008.

20. J. W. Salisbury and D. M. D'Aria, "Emissivity of terrestrial material in the 8-14 $\mu \mathrm{m}$ atmospheric window," Remote Sens. Environ., vol. 42, no. 2, pp. 83-106, Nov. 1992.

21. A. Heymsfield, J. S. Matrosov, and B. Baum, "Ice water path-optical depth relationships for cirrus and deep stratiform ice cloud layers," J. Appl. Meteor., vol. 42, no. 10, pp. 1369-1390, Oct. 2003.

22. D. K. Zhou, W. L. Smith, X. Liu, A. M. Larar, H.-L. Huang, J. Li, M. J. McGill, and S. A. Mango, "Thermodynamic and cloud parameters retrieval using infrared spectral data," Geophys. Res. Lett., vol. 32, no. L15, pp. 805.1-805.5, Aug. 2005.

23. D. K. Zhou, W. L. Smith, A. M. Larar, X. Liu, J. P. Taylor, P. Schlüssel, L. L. Strow, and S. A. Mango, “All weather IASI single field-of-view retrievals: Case study - Validation with JAIVEx data," Atmos. Chem. Phys., vol. 9, pp. 2241-2255, Mar. 2009. 